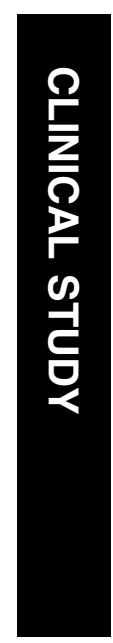

\title{
Microperimetric changes in neovascular age-related macular degeneration treated with ranibizumab
}

${ }^{1}$ Department of Ophthalmology, Queen's Medical Centre, Nottingham University Hospitals, Nottingham, UK

${ }^{2}$ Division of Ophthalmology and Vision Sciences, University of Nottingham, Queen's Medical Centre, Nottingham, UK

${ }^{3}$ MRC Lifecourse Epidemiology Unit, University of Southampton, Southampton General Hospital, Southampton, UK

Correspondence: W Amoaku, Division of Ophthalmology and Vision Sciences, University of Nottingham, Queen's Medical Centre, Nottingham, Nottinghamshire, NG7 2UH, UK

Tel: + 44 (0)1159249924 ext 62537;

Fax: +44 (0)1159709709

E-mail:wma@

nottingham.ac.uk

Received: 6 September 2011

Accepted in revised form: 17 December 2011

Published online:

10 February 2012

Part of this work was presented as a poster at ARVO, Fort Lauderdale, 2011.

\begin{abstract}
Purpose To assess the value of microperimetry in eyes with neovascular age-related macular degeneration previously treated with ranibizumab and now in the maintenance phase of therapy.
\end{abstract}

Methods A total of 21 eyes (14 patients) were included. Microperimetry was performed using the Macular Integrity Assessment Device on at least three occasions for each eye. Intravitreal ranibizumab was administered if visual acuity (VA) or optical coherence tomography (OCT) showed signs of active disease.

Results Five eyes showed no change in VA or OCT findings, and required no intravitreal injections. In these eyes, mean threshold sensitivity (TS) decreased by $13 \%$ (paired $t$-test, $P=0.05$ ) during the study period, but fixation stability (FS) was unchanged. In all, 16 eyes showed signs of disease activity, and therefore required ranibizumab injections during the study. In these eyes, VA, central retinal thickness (CRT), FS, and TS remained unchanged during follow-up. Peak TS was noted when CRT was $210 \mu \mathrm{m}$; above or below $210 \mu \mathrm{m}$, there was a gradual reduction in TS. Conclusion This study has provided novel information on the relationship between macular sensitivity, CRT, and VA in the maintenance phase of ranibizumab therapy. Patients with stable VA and CRT may still have deteriorating retinal sensitivity. This is usually a late manifestation and may indicate subclinical $\mathrm{CNV}$ activity.

Eye (2012) 26, 678-683; doi:10.1038/eye.2012.7; published online 10 February 2012

Keywords: microperimetry; wet macular degeneration; choroidal neovascular membrane
P Alexander ${ }^{1}$, F Mushtaq $^{2}$, C Osmond $^{3}$ and W Amoaku ${ }^{2}$

\section{Introduction}

Age-related macular degeneration (AMD) is the leading cause of blindness and visual disability in the western world. ${ }^{1}$ Neovascular AMD (nvAMD) accounts for approximately $75 \%$ of all cases of severe visual impairment due to AMD. ${ }^{2}$ Treatment outcomes have been improved considerably with the introduction of intravitreal therapies to inhibit vascular endothelial growth factor (VEGF), the central mediator of neovascularisation in wet AMD. Most clinical anti-VEGF therapy regimens include initiation with three consecutive monthly injections followed by a maintenance phase, during which injections are repeated pro re nata based on clinical findings. Clinical assessment would typically include visual acuity (VA), fundal examination, and optical coherence tomography (OCT) at monthly follow-up intervals. ${ }^{3}$ However, it is generally accepted that VA change or OCT alone may not be the optimum parameter(s) for measuring visual function in AMD.

Perimetry examines the sensitivity of different spatial locations of the retina, and is dependant on the retinal morphology. ${ }^{4}$ Microperimetry assesses the sensitivity of the central retina and allows precise topographic correlations of macular anatomy and light sensitivity. The value of such evaluations with the MP-1 microperimeter (Nidek, Gamagori, Japan) has been previously reported in AMD. ${ }^{5-8}$ These non-invasive measurements allow an automated analysis of macular function with appropriate correction for eye movements, even when fixation is unstable. As such, the assessments are independent of fixation and eye movements. The different retinal locations are 
mapped exactly in order to allow for subsequent comparisons. 7

The Macular Integrity Assessment Device (MAIA) system, a novel device that utilises scanning laser ophthalmoscope technology, is an advancement on the MP-1 device and has recently been introduced into clinical practice. MAIA is a third generation microperimetry system that measures visual sensitivity and fixation stability (FS) using 61 or 37 Goldmann style stimulus points within a 10-degree meridional grid centred on the fovea. It uses an automated eye tracking system that corrects 25 times per second to compensate for eye movements and/or poor fixation.

The MAIA has a maximum stimulus luminance of $1000 \mathrm{asb}$, and the stimulus dynamic range is $36 \mathrm{~dB}$ compared with the Nidek MP1, which has a maximum luminance of $400 \mathrm{asb}$ for a dynamic range of $20 \mathrm{~dB}$. The average visual sensitivity measured is compared with that in normals. ${ }^{9}$ It is fast, can be customised, and allows follow-up assessments.

The purposes of this pilot study are to evaluate MAIA microperimetry in routine clinical practice, to provide a preliminary assessment of its value in monitoring progress in eyes that are in the maintenance phase of treatment for nvAMD, and assess any potential correlations with VA and OCT measurements.

\section{Materials and methods}

Patients attending the AMD Clinic for treatment of one or both eyes were included in this study after obtaining informed consent. The period of study was from February 2010 to August 2010. All patients had previously been treated with intravitreal ranibizumab and were in the maintenance phase of therapy. Eyes with significant media opacities (whether corneal, lenticular, or vitreous) that interfered with OCT or clinical evaluation were excluded. In patients who had unilateral nvAMD, the fellow eye was excluded.

All patients had a routine clinical evaluation that included best corrected VA (LogMAR at $4 \mathrm{~m}$ ), slit lamp biomicroscopy of the anterior and posterior segments, 3D OCT (Topcon, Tokyo, Japan), and microperimetry (MAIA, CenterVue S.p.A, Padova, Italy). Intravitreal ranibizumab was subsequently administered as clinically necessary, as recommended by the Royal College of Ophthalmologists guidelines. ${ }^{3}$ Follow-up appointments were arranged monthly, where the same evaluations were repeated. For this report, patients were included only if they had at least three microperimetry measurements repeated at minimum intervals of 1 month or more.
In brief, for the microperimetry, patient identification details, age, date of examination, examination number, and type of examination (baseline, follow-up and so on) were entered into the database. The measurements were then obtained for each eye. Data were analysed statistically with the dedicated MAIA software, the EYEdB (CenterVue S.p.A), which utilises a normative database to correlate the average threshold, FS, and patient's age into a macular integrity (MI) index.

Average threshold was defined as the arithmetic mean of all responses, in decibels $(\mathrm{dB}) .0 \mathrm{~dB}$ means that the patient was only able to see the maximum stimuli intensity (1000 asb). When patients cannot see that maximum intensity, then MAIA evaluates it with the symbol '\#'.

FS indices, P1 and P2, were defined as the percentage of fixation points falling inside a circle of $1^{\circ}$ and $2^{\circ}$ radius, respectively. Eyes with more than $75 \%$ of the fixation points located within a $2^{\circ}$ diameter circle positioned with respect to the gravitational centre of all fixation points $(\mathrm{P} 1>75 \%)$ are classified as stable. If $<75 \%$ of the fixation points are located within a $2^{\circ}$ diameter circle, but more than $75 \%$ of the fixation points are located within a $4^{\circ}$ diameter circle positioned with respect to the gravitational centre of all fixation points (P1 $<75 \%$, P2 $>75 \%$ ), fixation is classified as relatively unstable. If $<75 \%$ of fixation points are located within $4^{\circ}$ diameter circle positioned with respect to the gravitational centre of all fixation points (P1 and P2 $<75 \%$ ), the pattern is described as being unstable fixation.

MI, a global index of sensitivity, was calculated by incorporating the threshold and FS indices. MI has been developed using a neural network trained on over 800 eyes to recognise normal sensitivity patterns (low index values) and abnormal ones (high index values).

For each eye, the baseline and follow-up examinations were evaluated, and a differential analysis generated for the FS indices, individual threshold in $\mathrm{dB}$ for each stimulus point, and the average threshold for each visit.

The Institutional Review Board has granted this project exemption from formal ethical approval.

\section{Statistical analysis}

Paired $t$-tests (two-tailed) were used to compare measurements of VA, central retinal thickness, threshold sensitivity (TS), and fixation sensitivity between the clinically stable and unstable patients, between the start and end of the follow-up period. With our current design and a sample size of 21 eyes, and using a test at the $5 \%$ level of statistical significance, we had $88 \%$ power to detect a difference of $3 \mathrm{~dB}$ in the sensitivity change 
Table 1 Comparison of visual acuity, OCT, and microperimetry parameters in eyes deemed 'unstable' and therefore requiring further intravitreal ranibizumab, and in 'stable' eyes that required no treatment during the follow-up period

\begin{tabular}{|c|c|c|c|c|}
\hline \multirow[b]{2}{*}{ Number } & \multicolumn{2}{|c|}{ Unstable eyes } & \multicolumn{2}{|c|}{ Stable eyes } \\
\hline & 16 eyes & & 5 eyes & \\
\hline Change in VA & +3 letters & Not significant & 0 letters & Not significant \\
\hline Change in CRT & $-12 \mu \mathrm{m}$ & Not significant & $+19 \mu \mathrm{m}$ & Not significant \\
\hline Change in TS & $-0.9 \%$ & Not significant & $-13 \%$ & $P=0.05$ \\
\hline Change in fixation stability & $+0.01 \%$ & Not significant & $-19.9 \%$ & $P=0.14$ \\
\hline
\end{tabular}

when comparing the clinically stable and unstable groups.

\section{Results}

A total of 21 eyes of 14 patients with nvAMD were included in the study. Of the 14 patients, 6 (9 eyes) were male and 8 (12 eyes) were female. Patients' ages ranged from 52 to 86 (mean 72.9, SD 8.9). A total of 70 assessments were made; all patients were assessed at least three times during the follow-up period. As a result of the clinical assessment, intravitreal ranibizumab injections were administered in 28 of the 70 assessments $(40 \%)$. During the follow-up period, no endophthalmitis, progression of lens opacity, or other injection-related complications occurred.

Mean VA was 64 letters (range 33-85; SD 13) at the start of follow-up. Mean TS at the beginning of follow-up was $16.7 \mathrm{~dB}$ (SD 4.9). Mean FS was 83\% (SD 19, range 31-100) for P1 and 96\% (SD 6, range 75-100) for P2. Therefore, 7 eyes had stable fixation (P1>75\%), and 14 eyes had relatively unstable fixation $(\mathrm{P} 1<75 \%$, $\mathrm{P} 2>75 \%)$. No eyes had unstable fixation. MI was calculated as 98 (SD 5, range 84-100). Mean central retinal thickness at the beginning of follow-up was $223 \mu \mathrm{m}$ (SD $49 \mu \mathrm{m})$.

During the period of follow-up, five eyes were deemed stable and required no intravitreal injections according to the treatment protocol (see Table 1). In these eyes, mean change in VA was $0 \operatorname{logMAR}$ letters (range -7 to +6 letters) over the follow-up period, with mean change in central retinal thickness of $+19 \mu \mathrm{m}$ (range -4 to +88 , SD 39 , not significant). However, mean TS decreased by $1.96 \mathrm{~dB}(P=0.05)$ during the follow-up period. A reduction in mean FS (73.6-58.2\%) was noted during the follow-up period, but this was not statistically significant $(P=0.14)$.

In all, 16 eyes showed signs of disease activity on clinical examination or OCT, and therefore required at least one intravitreal ranibizumab injection during the period of follow-up. In these eyes, mean change in VA during follow-up was a gain of $3 \log$ MAR letters (range +13 to -11 letters). The central retinal thickness

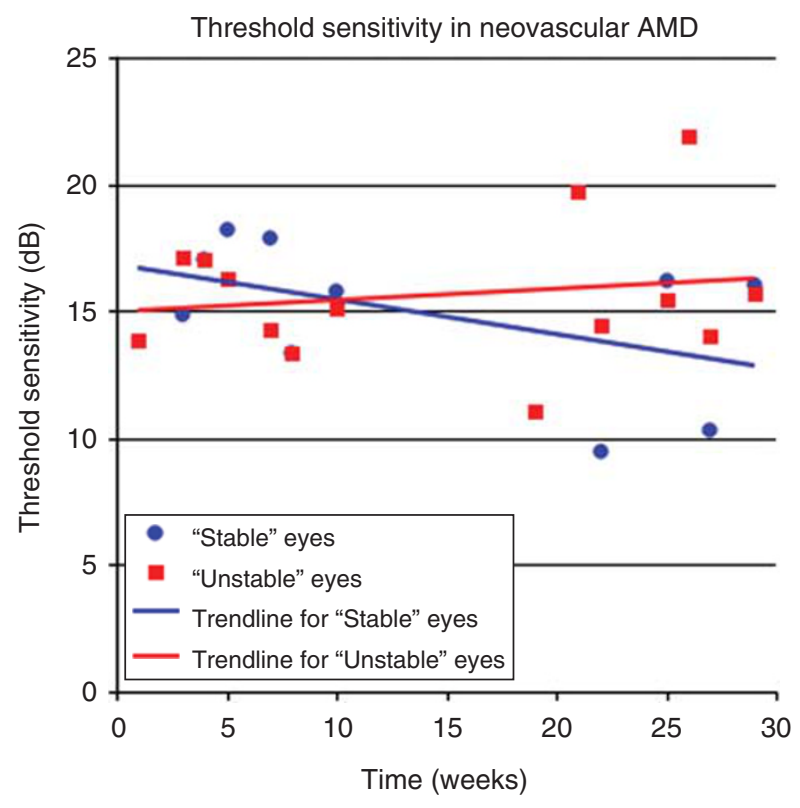

Figure 1 Change in TS over time in eyes deemed as 'unstable' and therefore requiring further intravitreal ranibizumab, and in 'stable' eyes that required no treatment during the follow-up period.

showed a mean change of $-12 \mu \mathrm{m}$ (range -113 to +38 , SD 37). Mean TS was almost unchanged during the follow-up period, showing a reduction of $0.16 \mathrm{~dB}$ (not statistically significant) (see Figure 1). Mean FS was also unchanged.

The change in TS between stable and unstable eyes was compared. Using the TS at the start of follow-up as the predictor and the sensitivity at the end of the study as the outcome allows control of any regression to the mean, and also allows the use of a binary predictor variable to capture the difference in effect sizes between stable and unstable groups. Controlling for TS at the start of the study, eyes in the stable group had a $2.0 \mathrm{~dB}$ (SE $0.96, P=0.05$ ) reduction in sensitivity during the follow-up period, compared with the eyes in the unstable group.

VA was found to be correlated with TS, albeit weakly $\left(r^{2}=0.3\right)$. There was no correlation between VA and FS 


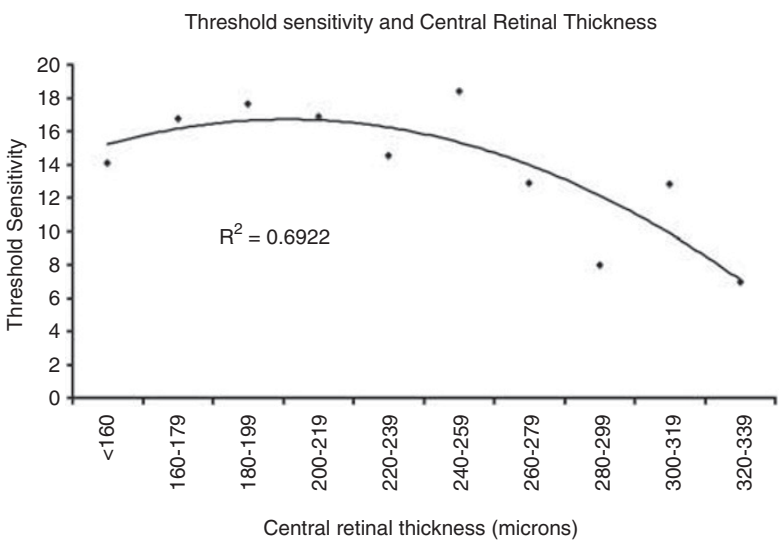

Figure 2 Change in TS (dB) with CRT $(\mu \mathrm{m})$.

indices P1 or P2. In this study, MI was not a useful indicator of disease, because it was very abnormal at baseline in almost all patients and not sensitive enough to detect changes between visits. Mean MI across all visits was 99.28 (SD 2.58).

Peak TS was noted when central retinal thickness (CRT) was $210 \mu \mathrm{m}$ (see Figure 2). Above $210 \mu \mathrm{m}$, there was a gradual reduction in TS that could be attributed to intraretinal and subretinal fluid. The reduction in TS associated with CRT $<210 \mu \mathrm{m}$ could be explained by a degree of photoreceptor atrophy.

\section{Discussion}

There are a number of psychophysical tests that can be used to quantify macular function, including VA, contrast sensitivity, photostress testing, and visual field sensitivity. Of these, VA remains the most important parameter to quantify the functional impact of AMD and to determine treatment decisions in individual patients.

Retinal sensitivity, as measured by microperimetry, may be more sensitive to changes in macular function because it assesses a larger retinal area than the conventional distance VA. ${ }^{10}$ The ability to perform activities of daily living are strongly dependant on the preservation of the central visual field. ${ }^{11}$ The size of central scotoma has already been established as an important determinant of reading capability, reading speed, and the ability to benefit from handheld magnifying lenses. ${ }^{12}$ Retinal sensitivity could be a better indicator of practical visual capabilities for patients with AMD.

Previous studies of microperimetry in AMD have included patients with dry AMD, ${ }^{13,14}$ and patients with nvAMD that have been treated with verteporfin photodynamic therapy, ${ }^{9}$ autologous RPE-choroid graft, ${ }^{15} 360$ degree macular translocation, ${ }^{16}$ systemic bevacizumab, ${ }^{17}$ intravitreal bevacizumab, ${ }^{8}$ and intravitreal ranibuzumab. ${ }^{18-20}$

Bolz et $a l^{18}$ conducted a prospective study to evaluate the functional and morphological effects of three loading doses of intravitreal ranibizumab in 29 patients (29 eyes) with previously untreated nvAMD. Although they observed an improvement in VA, central retinal thickness, and central retinal sensitivity after 1 month, they did not observe any additional benefit following the subsequent second and third injections. Their results led them to question whether three loading doses are mandatory in all patients or whether in some cases a single loading dose would be sufficient.

In a retrospective 24-month follow-up study, Parravano $e a^{20}$ used ranibizumab to treat 18 patients with nvAMD and noted that although VA and retinal thickness changes were maximal at 4 weeks after intravitreal ranibizumab, retinal sensitivity continued to show a trend for improvement. Although mean retinal sensitivity was maximal at 24 months, they did observe a slight fall in TS between the 6- and 12-month time points, but no explanation is given for this. Unlike in our study, no distinction was made between clinically stable patients and those with active disease requiring further ranibizumab injections. Lesion subtype was found to influence mean retinal sensitivity change between baseline and 24 months, with occult membranes producing a smaller response to treatment. The case mix may have influenced the differing response in our study. Of the 18 patients in Parravano's study, 2 showed a reduction in TS despite treatment with ranibizumab.

Prager $\mathrm{et} \mathrm{al}^{17}$ treated 14 patients (21 eyes) with systemic bevacizumab therapy, given as three initial infusions at 2-week intervals, followed by retreatment in the event of $\mathrm{CNV}$ recurrence. They noted that systemic bevacizumab induced a significant increase in mean retinal sensitivity, and a reduction in absolute scotoma size.

Ozdemir $\mathrm{et} \mathrm{al}^{8}$ found that intravitreal bevacizumab therapy in previously untreated patients induced a significant increase in mean retinal sensitivity and a reduction in mean scotoma size.

There are no previous studies in the literature on microperimetry in the maintenance phase of intravitreal anti-VEGF therapy for nvAMD. Our study documented and correlated functional and morphological changes in the maintenance phase of intravitreal ranibizumab therapy and found that all patients maintained VA over the study period. However, in patients who were unstable, and who therefore required additional ranibizumab injections during the course of the study, retinal sensitivity as measured by the MAIA was unchanged from baseline to the end of the study period. 
However, patients who were thought to be stable on routine clinical assessment, based on VA and OCT, showed a statistically significant deterioration in retinal sensitivity $(P=0.05)$.

It is possible that in patients who were stable on BCVA and OCT, there was an element of regression and fibrosis of the $\mathrm{CNV}$, which in turn could cause photoreceptor atrophy. This would be occurring at a rate too slow to be detected by changes in VA, but would be apparent on microperimetry testing. An alternative explanation is that in these supposedly stable patients, the CNV may still be active albeit at a subclinical level, and that the absence of anti-VEGF therapy led to a slow deterioration of retinal sensitivity. This supports the earlier report by Squirrel et $a l^{19}$ that microperimetry may provide more insight to changes in macular function compared to VA. In the recent CATT study, VA outcomes for monthly ranibizumab were found to be non-inferior to three loading doses of ranibizumab followed by re-treatment as required..$^{21}$ However, microperimetry was not performed in these patients and it would be interesting to know whether there is a difference in central retinal sensitivity and scotoma size between the two groups.

In a study of 43 patients (55 eyes), Landa et $a^{22}$ found that retinal sensitivity was correlated to the status of the underlying IS-OS junctional layer in both dry and wet forms of AMD. Loss of the IS-OS layer was significantly associated with poor retinal sensitivity, and compared with VA, functional testing with microperimetry appeared to correlate more consistently with changes in the outer retina, such as IS-OS junctional integrity, in patients with nvAMD.

Our finding that peak retinal sensitivity occurred with a CRT of $210 \mu \mathrm{m}$ is similar to findings of Hatef $e a^{23}$, who compared microperimetry findings with central retinal thickness in 21 eyes with diabetic macular oedema. Hatef et al showed that in subjects with a retinal thickness value of $280 \mu \mathrm{m}$ or less at the fovea, retinal thinning was associated with decreased retinal sensitivity. However, above $280 \mu \mathrm{m}$, retinal oedema was associated with decreased sensitivity. These data support the view that there is an optimal range of retinal thickness that correlates with optimal retinal sensitivity.

This pilot study has provided novel information on the relationship between macular sensitivity, retinal thickness, and VA in the maintenance phase of ranibizumab therapy for nvAMD. Patients may have stable VA and retinal thickness, but can still have deteriorating retinal sensitivity, suggesting that this is a late-stage manifestation of the disease. Additional studies on microperimetry in wet AMD are needed to determine whether repeated ranibizumab injections, even in stable patients, can help to prevent deterioration in central retinal sensitivity.

\section{Summary}

What was known before

- Microperimetry assesses the sensitivity of the central retina. It allows precise topographic correlations of macular anatomy and light sensitivity. Retinal sensitivity may be more sensitive to changes in macular function than the conventional distance visual acuity because it assesses a larger retinal area, and could be a better indicator of practical capabilities for patients with AMD. In treatment-naive wet AMD patients, anti-VEGF therapy induced a significant increase in mean retinal sensitivity and a reduction in mean scotoma size.

What this study adds

- This is the first study to assess microperimetry in the maintenance phase of intravitreal ranibizumab therapy for wet AMD. Patients with stable visual acuity and stable OCT parameters may still have deteriorating retinal sensitivity. This may indicate subclinical disease activity. Additional studies are needed to determine whether repeated anti-VEGF injections, even in 'stable' patients, can help to prevent deterioration in central retinal sensitivity.

\section{Conflict of interest}

The authors declare no conflict of interest.

\section{Acknowledgements}

We thank CenterVue for loaning the MAIA to us for a limited period.

\section{References}

1 Klein R, Klein BE, Jensen SC, Mares-Perlman JA, Cruickshanks KJ, Palta M. Age-related maculopathy in a multiracial United States population: the National Health and Nutrition Examination Survey III. Ophthalmology 1999; 106: 1056-1065.

2 Klein R, Klein BE, Jensen SC, Meuer SM. The five-year incidence and progression of age-related maculopathy: the Beaver Dam Eye Study. Ophthalmology 1997; 104: 7-21.

3 The Royal College of Ophthalmologists. Age-Related Macular Degeneration: Guidelines for Management. The Royal College of Ophthalmologists: London, 2009.

4 Lang G. Ophthalmology, 2nd ed. Thieme: New York, 2007.

5 Ritter M, Bolz M, Sacu S, Deák GG, Kiss C, Pruente C et al. Effect of intravitreal ranibizumab in avascular pigment epithelial detachment. Eye 2010; 24: 962-968.

6 Querques G, Forte R, Longo C, Carrillo P, Laculli C, Soubrane $\mathrm{G}$ et al. Microperimetry in age-related macular degeneration. J Fr Ophtalmol 2008; 31: 515-521.

7 Midena E, Radin PP, Pilotto E, Ghirlando A, Convento E, Varano M. Fixation pattern and macular sensitivity in eyes with subfoveal choroidal neovascularization secondary to age-related macular degeneration. A microperimetry study. Ophthalmology 2004; 19: 55-61. 
8 Ozdemir H, Karacorlu M, Senturk F, Karacorlu SA, Uysal O. Microperimetric changes after intravitreal bevacizumab injection for exudative age-related macular degeneration. Acta Ophthalmol 2012; 90: 71-75.

9 Midena E, Vujosevic S, Cavarzeran F. Normal values for fundus perimetry with the microperimeter MP1. Microperimetry Study Group. Ophthalmology 2010; 117: 1571-1576.

10 Okada K, Kubota-Taniai M, Kitahashi M, Baba T, Mitamura Y, Yamamoto S. Changes in visual function and thickness of macula after photodynamic therapy for age-related macular degeneration. Clin Ophthalmol 2009; 3: 483-488.

11 McClure ME, Hart PM, Jackson AJ, Stevenson MR, Chakravarthy U. Macular degeneration: do conventional measurements of impaired visual function equate with visual disability? Br J Opthalmol 2000; 84: 244-250.

12 Ergun E, Maar N, Radner W, Barbazetto I, Schmidt-Erfurth U, Stur M. Scotoma size and reading speed in patients with subfoveal occult choroidal neovascularization in age-related macular degeneration. Ophthalmology 2003; 110: 65-69.

13 Midena E, Vujosevic S, Convento E, Manfre A, Cavarzeran F, Pilotto E. Microperimetry and fundus autofluorescence in patients with early age-related macular degeneration. $\mathrm{Br} \mathrm{J}$ Ophthalmol 2007; 91: 1499-1503.

14 Hartmann KI, Bartsch DU, Cheng L, Kim JS, Gomez ML, Klein $\mathrm{H}$ et al. Scanning laser ophthalmoscope imaging stabilized microperimetry in dry age-related macular degeneration. Retina 2011; 31: 1323-1331.

15 Chen FK, Uppal GS, MacLaren RE, Coffey PJ, Rubin GS, Tufail A et al. Long-term visual and microperimetry outcomes following autologous retinal pigment epithelium choroid graft for neovascular age-related macular degeneration. Clin Experiment Ophthalmol 2009; 37: 275-285.

16 Uppal G, Feely M, Crossland M, Membrey L, Lee J, da Cruz L et al. Assessment of Reading Behaviour with an Infrared
Eyetracker following 360 Degree Macular Translocation for Age Related Macular Degeneration. Invest Ophthalmol Vis Sci 2011; 52(9): 6486-6496.

17 Prager F, Michels S, Simader C, Geitzenauer W, SchmidtErfurth U. Changes in retinal sensitivity in patients with neovascular age-related macular degeneration after systemic bevacizumab (avastin) therapy. Retina 2008; 28 : 682-688.

18 Bolz M, Simader C, Ritter M, Ahlers C, Benesch T, Prünte C et al. Morphological and functional analysis of the loading regimen with intravitreal ranibizumab in neovascular agerelated macular degeneration. Br J Ophthalmol 2010; 94: 185189.

19 Squirrel DM, Mawer NP, Mody CH, Brand CS. Visual outcome after intravitreal ranibizumab for wet age-related macular degeneration: a comparison between best-corrected visual acuity and microperimetry. Retina 2010; 30: 436-442.

20 Parravano M, Oddone F, Tedeschi M, Chiaravalloti A, Perillo L, Boccassini B et al. Retinal functional changes measured by microperimetry in neovascular age-related macular degeneration treated with ranibizumab: 24-month results. Retina 2010; 30: 1017-1024.

21 CATT Research Group Martin DF, Maguire MG, Ying GS, Grunwald JE, Fine SL, Jaffe GJ. Ranibizumab and bevacizumab for neovascular age-related macular degeneration. N Engl J Med 2011; 364: 1897-1908.

22 Landa G, Su E, Garcia PM, Seiple WH, Rosen RB. Inner segment-outer segment junctional layer integrity and corresponding retinal sensitivity in dry and wet forms of age-related macular degeneration. Retina 2011; 31: 364-370.

23 Hatef E, Colantuoni E, Wang J, Ibrahim M, Shulman M, Adhi $\mathrm{F}$ et al. The relationship between macular sensitivity and retinal thickness in eyes with diabetic macular edema. Am J Ophthalmol 2011; 152: 400-405. 